\title{
Aportes metodológicos para la medición del sentido de eficacia politica: Evidencia empirica de Quito-Ecuador
}

\author{
Methodological contributions for the measurement of political \\ efficacy sense: Empirical evidence from Quito-Ecuador
}

\author{
Marcos Zumárraga-Espinosa \\ Universidad Politécnica Salesiana, Ecuador \\ mzumarraga@ups.edu.ec (ECUADOR)
}

Recibido: 21.032018

Aceptado: 13.12.2019

\section{RESUMEN}

El sentido de eficacia política constituye un concepto explicativo fundamental para el entendimiento del repertorio de conductas políticas que tienen lugar en contextos democráticos. El presente trabajo tiene el propósito de contribuir metodológicamente a la medición del sentido de eficacia política, para lo cual propone un instrumento aplicable en entornos hispanoparlantes, tomando como punto de partida la evidencia empírica de su desempeño psicométrico en el caso ecuatoriano. Esta escala de eficacia política cuenta con dos componentes o subescalas: a) la adaptación y traducción de la escala de eficacia política interna (4 ítems) formulada por Niemi, Craig y Mattei (1991), y b) el desarrollo de 4 ítems para la medición de la eficacia política externa de los individuos, tomando como referencia la literatura disponible sobre el tema. Para la evaluación de las propiedades psicométrica del instrumento propuesto se emplearon dos muestras no probabilísticas tomadas antes $\left(\mathrm{n} \_1=1136\right)$ y después $\left(\mathrm{n} \_2=540\right)$ de las elecciones presidenciales ecuatorianas celebradas durante el año 2017. Los resultados más relevantes del estudio son los siguientes: primero, las pruebas de validez de constructo, mediante Análisis Factorial Confirmatorio (AFC), permiten verificar la bidimensionalidad de la escala, siendo capaz de medir de forma diferenciada la eficacia política interna y externa. Segundo, la confiabilidad de cada subescala cuenta con valores estadísticamente satisfactorios. Tercero, las pruebas de validez externa corroboran las expectativas teóricas relativas a la existencia de patrones de asociación distintos entre cada dimensión del sentido de eficacia política y otras variables de interés para la investigación sociopolítica. Cuarto, el 
análisis de invarianza de medida del instrumento, efectuado a través de Análisis Factorial Confirmatorio Multigrupo (AFCMG), sugiere la existencia de invarianza factorial, lo cual significa que los ítems del instrumento son capaces de realizar la misma distinción entre eficacia interna y externa en las dos muestras analizadas, además de ser interpretados de forma idéntica por los participantes de cada grupo. Finalmente, se discuten las implicaciones y limitaciones de los resultados obtenidos, así como las recomendaciones para futuros esfuerzos de investigación que permitan mejorar el ajuste de la escala propuesta al conjunto de países hispanoparlantes.

\section{PALABRAS CLAVE}

Eficacia política, eficacia política interna, eficacia política externa, medición, Ecuador.

\section{ABSTRACT}

Political efficacy sense constitutes a fundamental explanatory concept for understanding the repertoire of political behaviors that take place in democratic contexts. The purpose of this work is to contribute methodologically to the measurement of political efficacy sense, for which it proposes an applicable instrument for Spanish-speaking environments, taking as a starting point the empirical evidence of its psychometric performance in the Ecuadorian case. This scale of political efficacy has two components or subscales: a) the adaptation and translation of the internal political efficacy scale (4 items) developed by Niemi, Craig and Mattei (1991), and b) the development of 4 items for the measurement of the external political efficacy of the individuals, taking as reference the available literature about this subject. For the evaluation of the psychometric properties of the proposed instrument, two non-probabilistic samples were used, same as were taken before $\left(n \_1=1136\right)$ and after $\left(n \_2=540\right)$ of the Ecuadorian presidential elections held during the year 2017. The most relevant results of the study are the following: first, the tests of construct validity, through confirmatory factor analysis (CFA), allow to verify the two-dimensional structure of the scale, being able to measure differently the internal and external political efficacy. Second, the reliability of each subscale has statistically satisfactory values. Third, tests of external validity corroborate theoretical expectations relative to the existence of different association patterns between each dimension of political efficacy sense and other interest variables for sociopolitical research. Fourth, the measurement invariance analysis of the instrument, performed through Multi-Group Confirmatory Factor Analysis (MGCFA), suggests the existence of factorial invariance, which means that the items of the instrument are capable of making the same distinction between internal and external efficacy in the two analyzed samples, in addition they are interpreted identically by the participants of each 
group. Finally, the implications and limitations of the obtained results, as well as the recommendations for future research efforts to improve the adjustment of the proposed scale to the set of Spanish-speaking countries are in discussion.

\section{KEY WORDS}

Political efficacy, internal political efficacy, external political efficacy, measurement, Ecuador.

\section{INTRODUCCIÓN}

En el marco de la ciencia política, el sentido de eficacia política se ha mantenido hasta la actualidad como un concepto explicativo relevante para la comprensión de un amplio repertorio de conductas políticas que pueden tener lugar en entornos democráticos. Según la formulación inicial propuesta por Campbell, Gurin y Miller (1954), el sentido de eficacia política se define como aquel sentimiento relacionado con la posibilidad de ejercer un impacto en el proceso político a través de la acción política individual. Es decir, la medida en que una persona siente que puede influir a través de sus acciones sobre las decisiones tomadas por los representantes políticos o las autoridades públicas. La literatura disponible ha contribuido con evidencia empírica sobre el rol movilizador que la eficacia política ejerce en la adopción de acciones de participación política por parte de la ciudadanía. Este efecto movilizador se encuentra presente en el caso de actividades políticas de tipo convencional (Balch, 1974; Helal y Hamza, 2015; Jennings y Zeitner, 2003; Jiang, 2016; Scheufele y Nisbet, 2002; Valentino, Brader, Groenendyk, Gregorowicz y Hutchings, 2011; Zhang, Johnson, Seltzer y Bichard, 2010; Zumárraga Espinosa, Reyes Valenzuela y Carofilis Cedeño, 2017), al igual que cuando se trata de participación política a través del internet (Anduiza, Cantijoch, Gallego y Salcedo, 2010; Gil de Zúñiga, Molyneux y Zheng, 2014; Jiang, 2016; Yang y Dehart, 2016; Zumárraga Espinosa, Carofilis Cedeño y Reyes Valenzuela, 2018). Asimismo, se ha podido evidenciar que aquellos países que cuentan con un nivel de eficacia política más alto en su ciudadanía tienden a gozar de un mayor grado de participación política en general (Amnå, Munck y Zetterberg, 2004).

Aunque el sentido de eficacia política fue concebido originalmente como un constructo unidimensional, posteriores contribuciones permitieron distinguir entre dos dimensiones diferenciadas que integran este concepto: la eficacia política interna y la eficacia política externa (Acock y Clarke, 1990; Craig y Maggiotto, 1982; Craig, Niemi y Silver, 1990; Hayes y Bean, 1993). En este sentido, el grado de influencia que un individuo cree tener sobre los resultados producidos por el sistema político depende de dos perspectivas distintas aunque complementarias: la evaluación personal del sistema político o la autoevaluación de la propia competencia política. La eficacia política externa se centra en la evalu- 
ación individual del sistema político, razón por la cual engloba los sentimientos y creencias que una persona posee sobre el nivel de receptividad que el gobierno y las instituciones políticas tienen hacia las propuestas y demandas del sector social al que pertenece (Acock, Clarke y Stewart, 1985; Balch, 1974; Craig y Maggiotto, 1982; Kölln, Esaiasson y Turper, 2013). En términos generales, la eficacia política externa es una actitud individual relacionada con la observación del entorno político y la percepción de su grado de apertura hacia la participación de la ciudadanía, considerando la disponibilidad de canales y oportunidades para tal fin (Heiss y Matthes, 2016).

Por otra parte, tomando en cuenta las recientes aportaciones que, desde el campo de la psicología, ha realizado la teoría de la autoeficacia de Bandura (1997), la eficacia política interna puede definirse como el conjunto de creencias y sentimientos que el individuo posee sobre su propia capacidad para poner en marcha los cursos de acción necesarios para lograr objetivos propuestos en el campo de la política (Caprara, Vecchione, Capanna y Mebane, 2009; Vecchione et al., 2014). Esta conceptualización es compatible con la definición que comúnmente se ha venido empleando por la ciencia política, la cual concibe a la eficacia política interna como la autoevaluación que el individuo realiza sobre su capacidad para entender y participar satisfactoriamente en actividades de carácter político, tomando en cuenta los recursos, habilidades y conocimientos de los que dispone (Craig y Maggiotto, 1982; Finkel, 1985; Morrell, 2003; Niemi, Craig y Mattei, 1991).

En consecuencia, aunque la eficacia política interna y externa son componentes de un mismo constructo, cada una de estas dimensiones responde a determinantes distintos, razón por la cual tienden a comportarse de modo diferenciado aunque no totalmente independiente, pues se admite la posibilidad de que se correlacionen levemente (Acock y Clarke, 1990; Acock et al., 1985; Aish y Jöreskog, 1990; Craig y Maggiotto, 1982; Morrell, 2003). En otras palabras, dado que el sentido de eficacia política depende del objeto de actitud elegido, sea este el sistema político o el individuo autopercibido; un fuerte sentido de eficacia política interna no necesariamente coincidirá con un elevado grado de eficacia política externa. Mientras que la eficacia política externa depende en gran medida del contexto político, el cual es susceptible de modificarse entre procesos electorales, la eficacia política interna suele mantener un comportamiento más estable en el tiempo, debido a que responde a factores más sostenidos como el nivel de escolaridad, el estatus socioeconómico, el interés político o el conocimiento político (Acock y Clarke, 1990; Aish y Jöreskog, 1990; Balch, 1974; Craig y Maggiotto, 1982; Finkel, 1985; Hayes y Bean, 1993; McPherson, Welch y Clark, 1977; Morrell, 2003). Complementario a esto, recientes estudios han comenzado a incorporar la noción de eficacia política colectiva como un factor explicativo complementario para los fenómenos políticos, especialmente aquellos que involucran organizaciones (Caprara et al., 2009). No obstante, el presente estudio se enfocará en la eficacia política y sus dimensiones a escala individual. 


\subsection{La medición del sentido de eficacia política en entornos hispanoparlantes}

Una revisión del repertorio de investigaciones sobre comportamiento político efectuadas en América Latina y España permite identificar una falta de consenso metodológico para la medición del sentido de eficacia política. En este contexto, las formas en que se ha operacionalizado dicho concepto político pueden clasificarse en dos grandes estrategias de medición. El primer enfoque consiste en emplear las escalas de eficacia política desarrolladas ya sea por Campbell et al. (1954) o por Niemi et al. (1991), junto con una serie de adaptaciones, modificaciones y su respectiva traducción al español, procurando verificar la confiabilidad del instrumento en la mayoría de casos (Brussino, Medrano, Sorribas y Rabbia, 2011; Brussino, Rabbia y Sorribas, 2009; Grossi y Ovejero, 1994; Imhoff, Gutierrez y Brussino, 2009; Sorribas y Brussino, 2013). Las escalas e indicadores señalados tienden a emplearse de forma convencional en estudios políticos a nivel internacional. Un segundo enfoque ha optado por utilizar escalas propias creadas específicamente para una investigación dada, sin que exista el propósito de replicar tales modelos de medida en estudios posteriores, ni de verificar sus propiedades psicométricas (Anduiza et al., 2010; De Marco y Robles Morales, 2012; Somuano Ventura, 2005).

En general, se carece de un esfuerzo compartido por avanzar hacia una estandarización del modo de medición del sentido de eficacia política en entornos hispanoparlantes que, simultáneamente, asegure criterios de calidad psicométrica. Cuestión que puede distorsionar las conclusiones de aquellos estudios que, contemplando a la eficacia política como variable de interés, estén centrados en la identificación y contraste de predictores para diferentes actitudes y conductas políticas, dado que la correcta cuantificación de efectos depende de la calidad de las medidas utilizadas. Por otro lado, esta falta de estandarización produce dificultades para la comparación de los resultados obtenidos entre investigaciones efectuadas en diferentes contextos (Hayes y Bean, 1993; Morrell, 2003; Niemi et al., 1991).

El sentido de eficacia política es una derivación del concepto de autoeficacia en general, siendo un predictor fundamental para cualquier tipo de conducta, por lo tanto, la autoeficacia política es un factor explicativo clave para el entendimiento de cualquier comportamiento político individual (Caprara et al., 2009; Helal y Hamza, 2015; Vecchione et al., 2014). Esto refuerza la necesidad de una mayor dedicación metodológica a su medición, con el objetivo de dotar a la creciente investigación política de América Latina y España de un instrumento válido y confiable que contribuya a profundizar la comprensión de los fenómenos políticos ocurridos en estos contextos. Del mismo modo, se espera que este modelo de medición sea capaz de captar las diferencias entre el patrón de comportamiento de la dimensión interna y externa de la eficacia política.

Dados estos antecedentes, el objetivo del presente trabajo consiste en contribuir metodológicamente a la medición del sentido de eficacia política mediante la construcción de un instrumento que sea aplicable a contextos hispanoparlan- 
tes de forma válida y confiable. En este sentido, la adaptación y formulación de ítems para valorar la eficacia política interna y externa tomó como punto de partida la literatura disponible sobre el tema. La evaluación de las propiedades psicométricas de la escala de eficacia política desarrollada se realizó a través de tres etapas fundamentales: primero, se constató la bi-dimensionalidad de la escala y se examinó su validez de constructo mediante la aplicación de análisis factorial confirmatorio (AFC); segundo, se analizó la confiabilidad de la escala

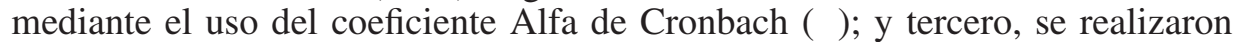
pruebas la validez externa para el instrumento, para lo cual se efectuó un análisis correlacional ( $\mathrm{r}$ de Pearson) entre las dimensiones de la eficacia política y una serie de variables sociodemográficas y políticas conceptualmente relacionadas. Finalmente, se puso a prueba la invarianza de medida de la escala de eficacia política entre dos muestras de estudio, la primera tomada en un momento previo y la segunda en un momento posterior a la segunda vuelta de las elecciones presidenciales ecuatorianas celebradas durante el año 2017.

\subsection{Una contextualización necesaria: el caso ecuatoriano}

Si bien este estudio propone una métrica que, con suficientes garantías psicométricas, pueda emplearse en el conjunto de países hispanoparlantes, no es menos cierto que el proceso de validación a reportarse se circunscribe al contexto político latinoamericano, en términos generales, y ecuatoriano, más específicamente. Por esta razón, resulta indispensable realizar una breve revisión contextual, a fin de aportar elementos que den cuenta de las particularidades que puedan emerger del análisis de datos, especialmente en la etapa de validación externa ${ }^{1}$.

A partir del año 2016, el panorama político de América Latina estuvo marcado por el agotamiento de un ciclo progresista que se había afianzado desde mediados de década del 2000 , teniendo como principales exponentes a países como Venezuela, Brasil, Argentina, Uruguay, Bolivia y Ecuador (Moreira, 2017). En el caso ecuatoriano, el proceso de la Revolución Ciudadana impulsado por el liderazgo carismático de Rafael Correa, desde su victoria presidencial en 2006, introdujo importantes cambios en el funcionamiento del sistema político y la conducción gubernamental de la política pública. En el plano político el periodo correísta se caracterizó por una inusitada estabilidad política y legitimidad electoral. Previo al 2006, Ecuador atravesaba una profunda crisis de representación política, al punto que no solo se generalizó una progresiva pérdida de confianza en los partidos políticos, sino hacia la política como un todo (Pachano, 2008). Cuestión que se acompañó de una continua inestabilidad política (desde 1996 se

${ }^{1}$ La validación externa en este trabajo se asume desde un enfoque de validez nomológica, que consiste en evaluar si las mediciones desarrolladas para la eficacia interna o externa se correlacionan empíricamente con otros constructos (criterios externos) que, según la literatura, se sabe que están relacionados (Batista-Foguet, Coenders y Alonso, 2004). 
habían tenido 7 presidentes en 10 años) y un escenario económico complicado (el país venía de una crisis bancaria que en 1998 causó la pérdida de la moneda nacional y una ola masiva de emigración). Tales antecedentes explicarían el apoyo popular mayoritario que obtuvo Correa en su ascenso a la presidencia, posicionándose como un outsider antiestablishment comprometido con la ciudadanización de la política (De la Cruz y Valenzuela, 2019). Con un discurso de tono populista, centrado en poner a la política al servicio de las grandes mayorías, Correa canalizó la insatisfacción y las reivindicaciones de amplios sectores de la población, organizaciones sociales y movimientos políticos de izquierda. La aprobación vía referéndum popular de la nueva constitución en 2008 y el fortalecimiento parlamentario de su partido Alianza País (AP), permitieron crear condiciones políticas favorables para la puesta en marcha del proyecto de la Revolución Ciudadana (Moreira, 2017). El sistema de partidos que previo a Correa se categorizaba como de pluralismo extremo, pasó a tomar la forma de un sistema de partido predominante (Pachano, 2008), con AP logrando mayorías parlamentarias en $2008(56.2 \%), 2009(43.5 \%)$ y 2013 (73.0\%). Esto, junto a las reformas institucionales de orden constitucional, posibilitó la concentración de poder en torno al ejecutivo y la figura del presidente (Meléndez y Moncagatta, 2017; Zapata-Mafla y Cuesta, 2018).

En el ámbito económico, el periodo correísta consolidó una política económica de corte redistributivo con énfasis en programas de inclusión y asistencia social, además de incrementar la inversión en obra pública, educación, salud y seguridad social. Especial mención merece la educación superior, entregándose en el periodo 2009-2013 un total de 14276 becas para estudios en el país y en el extranjero, en contraste con las 237 entregadas en el periodo 1995-2006 (Zapata-Mafla y Cuesta, 2018). Asimismo, los niveles de pobreza y desigualdad se redujeron considerablemente (Muñoz-Jaramillo, 2014). Como resultado, los avances sociales alcanzados configurarían una amplia base de apoyo popular para el sostenimiento electoral de la Revolución Ciudadana. Cabe mencionar que dicho modelo económico se sustentó en factores como los altos precios internacionales del petróleo, la estabilidad proporcionada por la dolarización y una gestión inicial inteligente del gobierno en aspectos como la renegociación de contratos petroleros y deuda externa, sin olvidar el apuntalamiento de la recaudación tributaria.

El 2017 (momento en que se realiza este estudio) constituyó un momento crucial para la continuidad del proyecto político de la Revolución Ciudadana, con unas elecciones presidenciales que, por primera vez desde 2006, no tenían a Correa como contendor. Después de no lograr un triunfo en primera vuelta, el 2 de abril se celebró el ballotage donde el candidato oficialista Lenin Moreno (ex vicepresidente de Rafael Correa), con apretado margen, se quedaría con la presidencia. El ambiente previo a estas elecciones estuvo cargado de acusaciones entre Moreno y el candidato opositor Guillermo Lasso (Partido CREO), tomando especial relevancia la trama internacional de corrupción relativa al "caso Odebrecht" que, a pesar de no revelarse nombres de involucrados para ese momento, presuntamente salpicaba a varias figuras importantes del oficialismo 
(Borja, 2017). Por su parte, el debilitamiento que venía mostrando AP desde las elecciones locales de 2014, quedó evidenciado con los resultados de las elecciones parlamentarias que acompañaron a la primera vuelta presidencial en febrero de 2017, pues aunque se obtuvo una mayoría parlamentaria absoluta, el número de escaños logrados se redujo en comparación con las elecciones de 2013 (Pachano, 2017). En consecuencia, el cambio de mando presidencial ocurrió en una coyuntura de desgaste para el proceso de la Revolución Ciudadana. Es así que a una situación económica que venía complicándose ${ }^{2}$ se sumó un cansancio importante de la ciudadanía en cuanto a las prácticas autoritarias que Correa había intensificado en sus últimos años de mandato, con retrocesos en términos de libertades y derechos, junto al deterioro de la institucionalidad democrática (Labarthe y Saint-Upéry, 2017; Meléndez y Moncagatta, 2017). Según cifras de Latinobarómetro (2016) la confianza en el gobierno pasó del 65\% en 2013 al $39 \%$ en 2016, y la satisfacción con el funcionamiento de la democracia pasó del $59 \%$ en 2013 al $41 \%$ en $2016^{3}$.

El panorama para el sostenimiento del proceso de la Revolución Ciudadana lucía entonces incierto, pues más allá del triunfo presidencial y parlamentario de AP, se debían afrontar dos problemas fundamentales: la ausencia del liderazgo personal de Correa y serios desequilibrios en las finanzas públicas. A partir de julio de 2017, Moreno comenzaría a distanciarse del correísmo, convocando al dialogo a todos los sectores de la sociedad (incluyendo los más fuertes opositores del anterior régimen), además de comprometerse con aspectos como el retorno a las prácticas democráticas, la lucha contra la corrupción y transparentar el balance económico de la década pasada. (Labarthe y Saint-Upéry, 2017). Posteriormente, este alejamiento del nuevo gobierno alcanzaría el punto de ruptura con la escisión de AP en un bloque correísta y otro morenista, el retorno del hacia el FMI y la adopción de un programa de austeridad fiscal. Añadiéndose además acusaciones de corrupción, excesos y malversaciones, con acciones judiciales de por medio, hacia una gran cantidad de figuras del núcleo duro correísta, incluyendo al mismo Correa asilado en Bélgica. Por último, las elecciones parlamentarias de 2017 mostraron señales de una nueva transición desde un sistema de partido predominante hacia un estado de mayor pluralismo, con un oficialismo en vías de fragmentación y una oposición heterogénea con partidos poco estructurados (Pachano, 2017).

${ }^{2}$ Desde el año 2014 la economía ecuatoriana comenzó a experimentar dificultades como la caída de los precios del petróleo, la apreciación del dólar y la reducción en el acceso a créditos internacionales, a lo que se sumó un fuerte sismo de 7.8 grados en 2016. En conjunto, estas condiciones adversas darían lugar a una desaceleración progresiva del crecimiento económico. Correa denominaría a tal convergencia desfavorable de factores como la "tormenta perfecta" (Álvarez, 2016).

${ }^{3}$ Para confianza en el gobierno se agrupan las respuestas: "mucha confianza" y "algo de confianza". La satisfacción con el funcionamiento de la democracia englobó las respuestas: "muy satisfecho" y "más bien satisfecho". 


\section{METODOLOGÍA}

\subsection{Participantes y recolección de datos}

La evaluación empírica de la métrica de eficacia política se efectuó a partir de dos muestras no probabilísticas de la población del Distrito Metropolitano de Quito (DMQ), Ecuador. La primera muestra $\left(n_{1}\right)$ estuvo conformada por 1136 participantes, conservando representatividad de la población quiteña en términos sociodemográficos. El $46.9 \%$ de esta muestra estuvo compuesta por hombres, mientras que el restante $53.1 \%$ por mujeres, la media de edad registrada fue de 34.3 años $(\mathrm{DT}=14.7)$. Con respecto al grado de escolaridad de los participantes, el $0.7 \%$ de la muestra no cuenta con instrucción formal, el $3.7 \%$ cuenta con educación primaria, el $8.2 \%$ tiene educación secundaria, un 45.2 cuenta con bachillerato, el $40.7 \%$ posee formación universitaria y el $1.5 \%$ ha realizado estudios de postgrado. El ingreso familiar mensual se evaluó a través de una escala con un intervalo mínimo 375 USD o menos (13.8\%), hasta un intervalo máximo de 2625 USD o más (4.6\%), la moda se situó en el segmento de "750 a 1124 USD" (29.1\%).

En la segunda muestra $\left(n_{2}\right)$ participaron 540 estudiantes universitarios, habiéndose recopilado información de 6 establecimientos universitarios situados en el DMQ. El porcentaje de participación de cada institución en esta muestra de estudio fue el siguiente: $37.5 \%$ de los estudiantes pertenece a la Universidad Central del Ecuador, 17.5\% a la Pontificia Universidad Católica del Ecuador, $12.5 \%$ a la Universidad de las Américas, $10.7 \%$ a la Universidad Politécnica Salesiana, $10.7 \%$ a la Escuela Politécnica Nacional y el $10.7 \%$ pertenece a la Universidad de las Fuerzas Armadas. Cada una de estas cuotas guarda correspondencia con el aporte relativo de cada establecimiento universitario a la población universitaria quiteña. El $49.6 \%$ de los participantes fueron mujeres y el 50.4\% hombres, la media de edad fue de 21.5 años $(D T=2.99)$. Los ingresos familiares mensuales se midieron con intervalos de valores que fluctúan entre 375 USD o menos $(8.9 \%)$ y 2625 USD o más (3.7\%), la moda se localizó en el intervalo de “750 a 1124 USD” (26.8\%).

La recolección de datos se efectuó mediante la aplicación de una encuesta general de opinión y participación política desarrollada por el Grupo de Investigaciones Psicosociales de la Universidad Politécnica Salesiana, Quito (GIPSUPS). La escala de eficacia política desarrollada formó parte del conjunto de variables que se midieron a partir de dicha encuesta. La primera aplicación de la encuesta se realizó durante el mes de marzo de 2017, previo a la segunda vuelta de las elecciones presidenciales ecuatorianas, mientras que la segunda aplicación se realizó en el mes de julio de 2017, posterior al mencionado proceso electoral. El levantamiento de información se efectúo a través de estudiantes de segundo nivel de la carrera de Psicología, quienes fueron previamente instruidos en el procedimiento de encuestado, enfatizando en la socialización del protocolo de confidencialidad y los propósitos de la investigación a los participantes. 
Los participantes de la primera muestra $\left(n_{1}\right)$ se seleccionaron por conveniencia, una vez establecidas las cuotas iniciales de edad y sexo. En la segunda muestra $\left(n_{2}\right)$, habiéndose planteado cuotas por institución universitaria, se efectuó una selección accidental, solicitando a los encuestadores que identifiquen lugares concurridos de cada campus para realizar aplicaciones en diferentes horarios. En cada caso se procuró que los participantes partan de una posición pasiva ante la encuesta (pudiendo decidir si realizarla o no), evitando así posibles sesgos de autoselección ${ }^{4}$.

\subsection{Instrumentos $\mathbf{y}$ mediciones}

Eficacia Política: El instrumento propuesto está compuesto por dos subescalas que buscan medir tanto la dimensión interna como externa del sentido de eficacia política. Para ello se realizó una adaptación de la escala propuesta por Niemi et al. (1991) en el caso de la eficacia política interna y se tomaron como referencia los trabajos elaborados por Aish y Jöreskog (1990), Acock y Clarke (1990), Amnå et al. (2004), Craig y Maggiotto (1982), Kölln et al. (2013) y McPherson et al. (1977) para la construcción de indicadores de eficacia política externa.

En el caso de la eficacia política interna los ítems empleados constituyen una adaptación de la escala de Niemi et al. (1991) a un entorno hispanoparlante. En este sentido, la traducción al español se realizó procurando conservar al máximo la esencia de la escala original, así como su orientación hacia la competencia política autopercibida por el individuo en términos de preparación, conocimientos y habilidades. Los ítems adaptados cuentan con una escala de valoración tipo Likert de 4 puntos: 4 (Totalmente de acuerdo), 3 (De acuerdo), 2 (En desacuerdo) y 1 (Totalmente en desacuerdo). En consecuencia, la escala de eficacia interna cuenta con 4 ítems que evalúan el grado de conformidad del individuo con las siguientes afirmaciones: "Siento que tengo una muy buena comprensión de las cuestiones políticas importantes que afronta nuestro país actualmente" (COMPRENSIÓN), "Me considero suficientemente preparado como para participar en política" (PREPARACIÓN), "Siento que puedo hacer tan buen trabajo en la función pública como la mayoría de la gente" (FUNCIÓN PÚBLICA), "Siento que estoy mejor informado sobre los asuntos del gobierno y la política que la mayoría de la gente" (INFORMACIÓN). El puntaje global de eficacia política interna es obtenido mediante la suma de los puntajes individuales de los ítems que integran la escala.

La eficacia política externa se midió a través de 4 ítems desarrollados con la finalidad de evaluar la medida en que el individuo percibe que el sistema político es receptivo y sensible ante las demandas y propuestas ciudadanas. Estos ítems

${ }^{4}$ Aunque este apartado describe la distribución por ingresos familiares de cada muestra, no se tomó en cuenta la clase social para selección de participantes 
registran el grado de conformidad del individuo con las siguientes afirmaciones: "El gobierno es cerrado e impide que gente como yo pueda participar en las decisiones que toma" (PARTICIPACIÓN), "Pienso que a los funcionarios públicos no les importa mucho lo que piense la gente como yo" (IMPORTANCIA), "En el Estado las autoridades siempre toman sus decisiones sin preocuparse por la opinión de la ciudadanía" (AUTORIDADES PÚBLICAS), "A los políticos les interesa solo los votos y no las opiniones de la gente" (POLÍTICOS). Al estar formulados de forma negativa, la escala de valoración tipo Likert de los indicadores propuestos es la siguiente: 1 (Totalmente de acuerdo), 2 (De acuerdo), 3 (En desacuerdo) y 4 (Totalmente en desacuerdo). El puntaje global de eficacia política externa se logra sumando los valores registrados por cada uno de los ítems que conforman la escala. La evaluación de la validez de contenido de los ítems de eficacia política externa estuvo a cargo de académicos especializados en el ámbito de la investigación sociopolítica.

Para la validación externa del instrumento se ha incluido un conjunto de variables que se encuentran conectadas conceptualmente y de manera diferenciada con cada dimensión del sentido de eficacia política. Se espera que la eficacia política interna se encuentre asociada positivamente con la educación, el interés en la política y la participación política convencional (Caprara et al., 2009; Morrell, 2003; Niemi et al., 1991). En contraste, la eficacia política externa se vincula de forma positiva con variables como la confianza en el gobierno y la satisfacción con la democracia (Acock et al., 1985; Craig y Maggiotto, 1982; Kölln et al., 2013).

Educación ${ }^{5}$ : El grado de escolaridad se evaluó a través de una escala que cuenta con las siguientes opciones de respuesta: 1 (Sin instrucción formal), 2 (Primera incompleta), 3 (Primaria completa), 4 (Secundaria incompleta), 5 (Secundaria terminada), 6 (Bachillerato incompleto), 7 (Bachillerato terminado), 8 (Universidad incompleta), 9 (Universidad terminada con título) y 10 (Postgrado).

Interés en la Política: Se midió a través de la pregunta: ¿Qué tan interesado diría Ud. que está en la política usualmente? La escala de valoración empleada oscila desde 1 (Nada interesado) hasta 4 (Muy interesado).

Participación Política Convencional: La evaluación de esta variable se realizó a partir de un índice aditivo compuesto por 8 actividades políticas de tipo convencional en las que los individuos pueden participar. Estas actividades van desde firmar peticiones, contactar con figuras políticas o funcionarios públicos, donar dinero con fines políticos, hasta participar en marchas y movilizaciones colectivas de carácter legal y pacífico. El grado de involucramiento en cada una de estas actividades se midió a través de una escala de valoración de 5 puntos, que va desde 1 (Nunca lo he hecho y nunca lo haría bajo ninguna circunstancia)

${ }^{5}$ La variable educación será empleada como criterio de validación externa solamente en el caso de la primera muestra. La segunda muestra, al centrarse en estudiantes universitarios, posee escasa variabilidad en el nivel de educación de los participantes, dificultando el análisis de correlación. 
hasta 5 (Lo he hecho muchas veces). El índice global de participación política convencional es el resultado de la sumatoria de los puntajes obtenidos en cada una de las actividades consultadas. Esta escala se elaboró tomando como referencia los trabajos de Ribeiro, Borba y Salinas (2011) y Somuano Ventura (2005). El grado de consistencia interna resultante es satisfactorio $\left(n_{1}: \alpha=0.83\right.$, $n_{2}: \alpha=0.88$ ).

Confianza en el Gobierno: Se evaluó a través de la pregunta: ¿Cuánta confianza tiene en el gobierno actual? Las opciones de respuesta oscilan desde 1 (Nada) hasta 4 (Mucha).

Satisfacción con la Democracia: Se midió a través de la pregunta: ¿Qué tan democrático es el gobierno hoy en día? La escala de valoración empleada varía desde 1 (No es nada democrático) hasta 5 (Completamente democrático).

\section{RESULTADOS}

Con el propósito de incrementar la rigurosidad del proceso de evaluación empírica de la escala de eficacia política desarrollada, en el presente trabajo se optó por emplear dos muestras independientes $\left(n_{1}\right.$ y $\left.n_{2}\right)$ tomadas en el contexto del DMQ. De esta manera, es posible verificar el grado de replicabilidad de las propiedades de validez y confiabilidad del instrumento al interior de un mismo contexto. Esto constituye un primer paso hacia la realización de contrastes empíricos de mayor alcance, sea a escala nacional o internacional.

\section{a) Validez de constructo y dimensiones de la escala de eficacia política}

Para la evaluación de la composición dimensional del instrumento se empleó la técnica de Análisis Factorial Confirmatorio (AFC). Esta técnica permite probar empíricamente un modelo factorial hipotético, en el cual se propone una cantidad concreta de factores o dimensiones que se relacionan con un conjunto de variables observables bajo ciertas especificaciones (Batista-Foguet et al., 2004; Lloret-Segura, Ferreres-Traver, Hernández-Baeza y Tomás-Marco, 2014). En otras palabras, mediante el AFC es posible comprobar en qué medida los datos se adecúan a las relaciones planteadas a nivel teórico entre los ítems de una escala y las respectivas dimensiones que deberían reflejar, además de posibles conexiones entre factores.

Dado que conceptualmente el sentido de eficacia política cuenta con una dimensión interna y otra externa, se esperaría que un modelo bidimensional tenga un mejor ajuste a los datos que un modelo unidimensional, el cual niega tal distinción entre eficacia política interna y externa. Como se puede observar en la figura 1, el modelo bidimensional asume que los ítems reflejan de forma diferenciada el comportamiento de la eficacia política interna y externa, admitiéndose una correlación entre estos factores. En contraste, el modelo unidimensional propone que todos los ítems son indicadores indistintos de un mismo factor común, asumiendo a la eficacia política como un concepto único. 
El AFC se efectuó mediante el paquete estadístico AMOS 23. Para la estimación de parámetros en los respectivos modelos se empleó el método de Distribución Libre Asintótica (ADF, del acrónimo en inglés: Asymptotically Distribution-Free), siendo equivalente al método de Mínimos Cuadrados Ponderados (Olsson, Foss y Troye, 2003). El método ADF es recomendable para datos correspondientes a variables categóricas que no cuentan con una distribución normal multivariante (Byrne, 2010; Clench-Aas, Nes, Dalgard y Aarø, 2011; Lloret-Segura et al., 2014). En el caso de los datos recolectados, la prueba de Mardia permitió concluir que los ítems de la escala de eficacia política no cuentan con una distribución normal multivariante, puesto que el coeficiente normalizado de Mardia arrojó un valor de 10.33, superando el máximo recomendado de 5 (Byrne, 2010). Del mismo modo, los ítems tipo Likert empleados para medir los dos tipos de eficacia política poseen escalas de valoración de 4 puntos, siendo recomendable considerar dichos ítems como variables categóricas, pues para suponer un mayor nivel de medición es necesario contar con al menos 5 categorías de respuesta (Clench-Aas et al., 2011; Lloret-Segura et al., 2014).

Figura 1. Estructuras factoriales alternativas para el constructo sentido de eficacia política

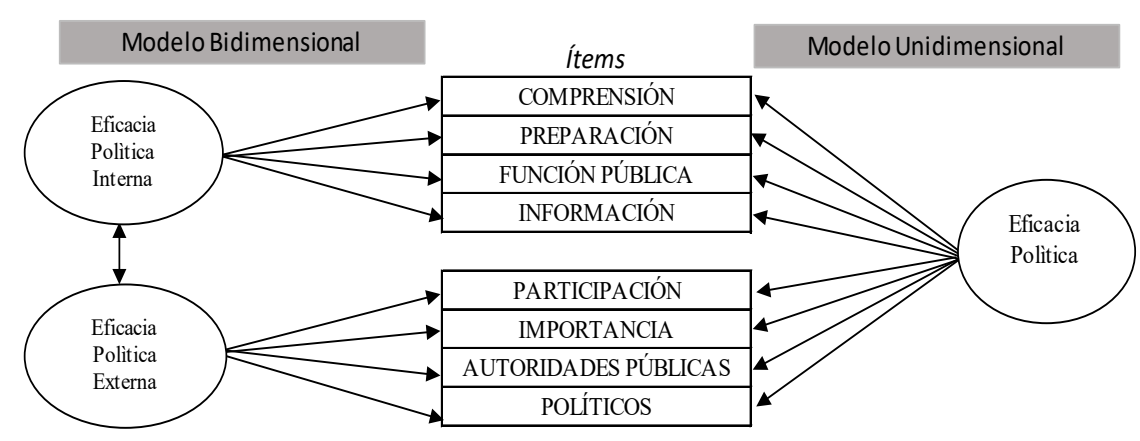

Elaboración propia.

Para la comparación entre modelos se consideraron los siguientes indicadores de bondad de ajuste: prueba chi-cuadrado ( $\mathrm{x} 2$ ), razón entre chi-cuadrado y el número de grados de libertad (X2/gl), índice de bondad de ajuste comparativo (CFI), índice de Tucker-Lewis (TLI), índice de ajuste normalizado (NFI), índice de bondad de ajuste corregido (AGFI), raíz del residuo cuadrático promedio de aproximación (RMSEA), el tamaño muestral crítico de Hoelter (CN) y el criterio de información de Akaike (AIC). Estos indicadores en su conjunto permiten evaluar que tan bien el modelo propuesto es capaz de reproducir la matriz de covarianzas observadas entre los ítems o variables que conforman el instrumento (Byrne, 2010; Ruiz, Pardo y San Martín, 2010). 
La hipótesis de partida de la prueba $\mathrm{X} 2$ consiste en suponer que la matriz de covarianzas reproducida por el modelo es equivalente a las covarianzas observadas, razón por la cual se espera un resultado no significativo $(>0.05)$. No obstante, la prueba $\mathrm{X} 2$ es altamente sensible al tamaño muestral, lo cual tiende a dificultar el logro del criterio de referencia mencionado cuando se trabaja con muestras grandes, es decir, superiores a 200 casos (Caprara et al., 2009; Ruiz et al., 2010; Schermelleh-Engel, Moosbrugger y Müller, 2003). A pesar de ello, disminuciones en el valor del estadístico $X^{2}$ pueden interpretarse como una mejoría en el ajuste de los datos al modelo propuesto (Cupani, 2012). Dadas las limitaciones de X2 la alternativa recomendada es el índice X2/gl, para el cual valores entre 1 y 3 implican un buen ajuste empírico del modelo (Miranda-Zapata, Riquelme-Mella, Cifuentes-Cid y Riquelme-Bravo, 2014; Ruiz et al., 2010; Yang y DeHart; 2016). Los valores de referencia para el resto de indicadores de bondad de ajuste son los siguientes: CFI, TLI y NFI $\geq 0.95$; AGFI $\geq 0.95$; RMSEA < 0.08 (Byrne, 2010; Milfont y Fischer, 2010; Milošević-Đorđević y Žeželj, 2017; Miranda-Zapata et al., 2010; Ruiz et al., 2010; Nuviala Nuviala et al., 2014).

El índice $\mathrm{CN}$ de Hoelter establece el tamaño muestral suficiente para aceptar la hipótesis de un ajuste perfecto de los datos a un nivel de significación determinado, valores por encima de 200 indican un buen ajuste (Byrne, 2010; Caprara et al., 2009; Liang, Asano, Bollen, Kahana y Maeda, 1987). Por último, el índice AIC se aplica comúnmente para fines comparativos, considerando que mientras más bajo sea el valor del mismo, mejor el ajuste del modelo (Schermelleh-Engel et al., 2003; Wagenmakers y Farrell, 2004). La tabla 1 presenta los resultados del AFC efectuado para cada muestra de estudio por separado.

Tabla 1. Comparación de estructura unidimensional vs bidimensional: Sentido de Eficacia Política

\begin{tabular}{|c|c|c|c|c|}
\hline \multirow{2}{*}{$\begin{array}{c}\text { Indicadores } \\
\text { de Bondad de } \\
\text { Ajuste }\end{array}$} & \multicolumn{2}{|c|}{$\begin{array}{c}n_{1}=1136 \text { (Población General } \\
\text { - Quito) }\end{array}$} & \multicolumn{2}{c|}{$\begin{array}{c}n_{2}=540 \text { (Estudiantes } \\
\text { Universitarios - Quito) }\end{array}$} \\
\cline { 2 - 5 } & $\begin{array}{c}\text { Modelo } \\
\text { Unidimensional }\end{array}$ & $\begin{array}{c}\text { Modelo } \\
\text { Bidimensional }\end{array}$ & $\begin{array}{c}\text { Modelo } \\
\text { Unidimensional }\end{array}$ & $\begin{array}{c}\text { Modelo } \\
\text { Bidimensional }\end{array}$ \\
\hline$X^{2}$ & 282.93 & 39.52 & 200.44 & 26.36 \\
\hline$g l$ & 20 & 17 & 20 & 17 \\
\hline $\begin{array}{c}p \text {-valor } \\
\text { Nivel de } \\
\text { Probabilidad) }\end{array}$ & 0.000 & 0.002 & 0.000 & 0.068 \\
\hline X2/gl & 14.15 & 2.33 & 10.02 & 1.55 \\
\hline CFI & 0.64 & 0.97 & 0.61 & 0.98 \\
\hline TLI & 0.50 & 0.95 & 0.45 & 0.97 \\
\hline
\end{tabular}




\begin{tabular}{|c|c|c|c|c|}
\hline NFI & 0.63 & 0.95 & 0.59 & 0.95 \\
\hline \multirow{2}{*}{$\begin{array}{c}\text { Indicadores } \\
\text { de Bondad de } \\
\text { Ajuste }\end{array}$} & \multicolumn{2}{|c|}{$n_{1}=1136$ (Población General } & \multicolumn{2}{c|}{$\begin{array}{c}n_{2}=540 \text { (Estudiantes } \\
\text { Universitarios - Quito) }\end{array}$} \\
\cline { 2 - 5 } & $\begin{array}{c}\text { Modelo Quito) } \\
\text { Unidimensional }\end{array}$ & $\begin{array}{c}\text { Modelo } \\
\text { Bidimensional }\end{array}$ & $\begin{array}{c}\text { Modelo } \\
\text { Unidimensional }\end{array}$ & $\begin{array}{c}\text { Modelo } \\
\text { Bidimensional }\end{array}$ \\
\hline AGFI & 0.81 & 0.97 & 0.81 & 0.97 \\
\hline RMSEA & 0.11 & 0.03 & 0.13 & 0.03 \\
\hline $\begin{array}{c}\text { CN de Hoelter } \\
\text { (0,01) }\end{array}$ & 151 & 960 & 102 & 684 \\
\hline AIC & 314.93 & 77.52 & 232.48 & 64.36 \\
\hline
\end{tabular}

Fuente: Datos recolectados por el GIPS-UPS, Quito - Ecuador (marzo 2017 y julio 2017). Elaboración propia.

En su conjunto, el comportamiento de los indicadores de bondad de ajuste permite confirmar que el modelo bidimensional se adecúa de mejor manera a los datos empíricos que el modelo unidimensional. Aunque la prueba $X 2$ resulta significativa en todos los casos, tanto en $n_{1}$ como $n_{2}$ el estadístico X2 sufre una reducción drástica cuando se pasa de un modelo unifactorial a un modelo con dos factores latentes, lo cual implica una mejoría del ajuste. Los restantes indicadores adoptan valores estadísticamente satisfactorios cuando se trata de un modelo bidimensional, mientras que no alcanzan los criterios de referencia en modelos con un solo factor latente. Estos resultados se repiten en las dos muestras analizadas $\left(n_{1}\right.$ y $\left.n_{2}\right)$.

Las cargas factoriales del modelo bidimensional concuerdan con el criterio recomendado de un valor mayor a 0.40 (Caprara et al., 2009; Hayes y Bean, 1993; Liang et al., 1987; Lloret-Segura et al., 2014), variando desde 0.69 hasta 0.86 en el caso de $n_{1}(M=0.76 ; D T=0.07)$, y desde 0.68 hasta 0.88 para $n_{2}(M$ $=0.76 ; D T=0.06)$. Todas las cargas factoriales son estadísticamente significativas (véase tabla 2). Complementariamente, en las soluciones factoriales duales la correlación entre factores latentes es débil tanto para $n_{1}$ como para $n_{2}(\mathrm{r}<$ 0.30 ), el signo negativo sugiere una asociación inversa entre la eficacia política interna y externa. 
Tabla 2. Modelo bidimensional del sentido de eficacia política: Cargas factoriales y correlación entre factores latentes

\begin{tabular}{|c|c|c|c|c|}
\hline \multirow{2}{*}{$\begin{array}{l}\text { Ítems de la Escala de } \\
\text { Eficacia Política }\end{array}$} & \multicolumn{2}{|c|}{$\begin{array}{c}n_{1}=1136 \text { (Población } \\
\text { General - Quito) }\end{array}$} & \multicolumn{2}{|c|}{$\begin{array}{l}n_{2}=540 \text { (Estudiantes } \\
\text { Universitarios - Quito) }\end{array}$} \\
\hline & $\begin{array}{l}\text { Factor } 1 \\
\text { Eficacia } \\
\text { Política } \\
\text { Interna }\end{array}$ & $\begin{array}{l}\text { Factor } \\
\text { Eficacia } \\
\text { Política } \\
\text { Externa }\end{array}$ & $\begin{array}{l}\text { Factor } 1 \\
\text { Eficacia } \\
\text { Política } \\
\text { Interna }\end{array}$ & $\begin{array}{ll} & \text { Factor } \\
2 & \text { Eficacia } \\
\text { Política } \\
\text { Externa }\end{array}$ \\
\hline $\begin{array}{l}\text { Siento que tengo } \\
\text { una muy buena } \\
\text { comprensión de las } \\
\text { cuestiones políticas } \\
\text { importantes que } \\
\text { afronta nuestro } \\
\text { país actualmente } \\
\text { (COMPRENSIÓN). }\end{array}$ & $0.70 * * *$ & & $0.68 * * *$ & \\
\hline $\begin{array}{l}\text { Me considero } \\
\text { suficientemente } \\
\text { preparado como para } \\
\text { participar en política } \\
\text { (PREPARACIÓN). }\end{array}$ & $0.69 * * *$ & & $0.76 * * *$ & \\
\hline $\begin{array}{l}\text { Siento que podría } \\
\text { hacer tan buen trabajo } \\
\text { en la función pública } \\
\text { como la mayoría de } \\
\text { la gente (FUNCIÓN } \\
\text { PUBLICA). }\end{array}$ & $0.69 * * *$ & & $0.77 * * *$ & \\
\hline $\begin{array}{l}\text { Siento que estoy mejor } \\
\text { informado sobre los } \\
\text { asuntos del gobierno } \\
\text { y la política que la } \\
\text { mayoría de la gente } \\
\text { (INFORMACIÓN). }\end{array}$ & $0.76 * * *$ & & $0.70 * * *$ & \\
\hline $\begin{array}{l}\text { El gobierno es cerrado } \\
\text { e impide que gente } \\
\text { como yo pueda } \\
\text { participar en las } \\
\text { decisiones que toma } \\
\text { (PARTICIPACIÓN). }\end{array}$ & & $0.77 * * *$ & & $0.72 * * *$ \\
\hline
\end{tabular}




\begin{tabular}{|c|c|c|c|c|}
\hline \multirow{2}{*}{$\begin{array}{l}\text { Ítems de la Escala de } \\
\text { Eficacia Política }\end{array}$} & \multicolumn{2}{|c|}{$\begin{array}{c}n_{1}=1136 \text { (Población } \\
\text { General - Quito) }\end{array}$} & \multicolumn{2}{|c|}{$\begin{array}{l}n_{2}=540 \text { (Estudiantes } \\
\text { Universitarios - Quito) }\end{array}$} \\
\hline & $\begin{array}{l}\text { Factor } 1 \\
\text { Eficacia } \\
\text { Politica } \\
\text { Interna }\end{array}$ & $\begin{array}{l}\text { Factor } 2 \\
\text { Eficacia } \\
\text { Política } \\
\text { Externa }\end{array}$ & $\begin{array}{l}\text { Factor } 1 \\
\text { Eficacia } \\
\text { Política } \\
\text { Interna }\end{array}$ & $\begin{array}{l}\text { Factor } 2 \\
\text { Eficacia } \\
\text { Política } \\
\text { Externa }\end{array}$ \\
\hline $\begin{array}{l}\text { Pienso que a los } \\
\text { funcionarios públicos } \\
\text { no les importa } \\
\text { mucho lo que piense } \\
\text { la gente como yo } \\
\text { (IMPORTANCIA). }\end{array}$ & & $0.86 * * *$ & & $0.88 * * *$ \\
\hline $\begin{array}{l}\text { En el Estado las } \\
\text { autoridades siempre } \\
\text { toman sus decisiones } \\
\text { sin preocuparse } \\
\text { por la opinión } \\
\text { de la ciudadanía } \\
\text { (AUTORIDADES } \\
\text { PÚBLICAS). }\end{array}$ & & $0.85 * * *$ & & $0.79 * * *$ \\
\hline $\begin{array}{l}\text { A los políticos } \\
\text { les interesa solo } \\
\text { los votos y no las } \\
\text { opiniones de la gente } \\
\text { (POLÍTICOS). }\end{array}$ & & $0.74 * * *$ & & $0.75^{*} * *$ \\
\hline $\begin{array}{l}\text { Correlación entre } \\
\text { Eficacia Política } \\
\text { Interna y Eficacia } \\
\text { Política Externa }(r)\end{array}$ & \multicolumn{2}{|c|}{$-0.27 * * *$} & \multicolumn{2}{|c|}{$-0.24 * * *$} \\
\hline
\end{tabular}

Nota: $* * * * p<0.001(0.1 \%)$. Los valores presentados en esta tabla corresponden a los coeficientes estandarizados estimados por AMOS 23 Fuente: Datos recolectados por el GIPS-UPS, Quito - Ecuador (marzo 2017 y julio 2017). Elaboración propia. 


\section{b) Análisis de Confiabilidad de la Escala de Eficacia Política}

Para evaluar la consistencia interna del modelo de medición propuesto se procedió a calcular el coeficiente Alfa de Cronbach $(\alpha)^{6}$ de las subescalas que miden tanto la eficacia política interna como externa. Asimismo, se obtuvo un coeficiente $\alpha$ global para todos los ítems que integran la escala de eficacia política, sin distinguir entre dimensiones. Los resultados del análisis de confiabilidad se presentan en la tabla 3 .

Tabla 3. Análisis de consistencia interna de la escala de eficacia política y sus componentes

\begin{tabular}{|c|c|c|}
\hline \multirow{2}{*}{ Escalas } & \multicolumn{2}{|c|}{$\begin{array}{l}\text { Alfa de Cronbach } \\
(\alpha)\end{array}$} \\
\hline & $\begin{array}{c}n_{1}=1136 \\
\text { (Población General - } \\
\text { Quito) }\end{array}$ & $\begin{array}{l}n_{2}=540 \text { (Estudiantes } \\
\text { Universitarios - Quito) }\end{array}$ \\
\hline $\begin{array}{l}\text { Subescala de Eficacia Política } \\
\text { Interna }\end{array}$ & 0.79 & 0.81 \\
\hline $\begin{array}{l}\text { Subescala de Eficacia Política } \\
\text { Externa }\end{array}$ & 0.88 & 0.86 \\
\hline $\begin{array}{l}\text { Escala de Eficacia Política } \\
\text { (Global) }\end{array}$ & 0.61 & 0.60 \\
\hline
\end{tabular}

Fuente: Datos recolectados por el GIPS-UPS, Quito - Ecuador (marzo 2017 y julio 2017). Elaboración propia.

Como se puede observar, las subescalas de eficacia política interna y externa presentan valores estadísticamente satisfactorios para el coeficiente $\alpha$, pues tanto para $n_{1}$ como $n_{2}$ los valores obtenidos son superiores a 0.70 , lo cual indica un buen nivel de consistencia interna (Oviedo y Arias, 2005; González Alonso y Pazmiño Santacruz, 2015). En consecuencia, tanto los ítems de eficacia política interna como externa generan mediciones que resultan altamente estables e intercambiables entre sí. Dicho de otro modo, los puntajes obtenidos en cada ítem tienden a ser similares a aquellos obtenidos en el resto de ítems de la misma subescala (BatistaFoguet et al., 2004; Oviedo y Arias, 2005). Adicionalmente, la contracción que experimenta el coeficiente $\alpha$ al considerar la escala de eficacia

${ }^{6}$ Con la finalidad de realizar una evaluación conservadora de la confiabilidad del instrumento se optó por emplear el coeficiente a convencional, puesto que aunque el coeficiente alfa ordinal sería apropiado dada la naturaleza politómica de los ítems, dicho coeficiente tiende a sobreestimar el nivel de confiabilidad (Elosua Oliden y Zumbo, 2008). De igual manera, no se recomienda el uso del alfa ordinal para extraer conclusiones de confiabilidad, puesto que es un indicador de confiabilidad hipotética (sustitución de ítems categóricos por un formato cuantitativo) antes que de confiabilidad observada (Chalmers, 2017). 
política de forma global coincide con los resultados obtenidos en el AFC, puesto que aunque exista una leve correlación entre la dimensión interna y externa de la eficacia política, estas tienden a presentar patrones distintos de comportamiento.

\section{c) Evaluación de validez externa}

Como criterio externo de validación se seleccionó un conjunto de variables políticas y sociodemográficas que se asocian con el sentido de eficacia política desde una perspectiva teórica. No obstante, dado que tanto la eficacia política interna como externa responden a determinantes distintos, se espera que se asocien de forma diferenciada con cada variable externa considerada. La tabla 4 muestra los resultados del análisis correlacional $^{7}$ efectuado para cada muestra de estudio.

Tabla 4. Correlaciones de Pearson entre tipos de eficacia política y criterios externos

\begin{tabular}{|c|c|c|c|c|}
\hline \multirow{2}{*}{$\begin{array}{c}\text { Criterios externos de } \\
\text { validación }\end{array}$} & \multicolumn{2}{|c|}{$\begin{array}{c}n_{1}=1136 \text { (Población } \\
\text { General - Quito) }\end{array}$} & \multicolumn{2}{c|}{$\begin{array}{c}n_{2}=540 \text { (Estudiantes } \\
\text { Universitarios - Quito) }\end{array}$} \\
\cline { 2 - 5 } & $\begin{array}{c}\text { Eficacia } \\
\text { Política } \\
\text { Interna }\end{array}$ & $\begin{array}{c}\text { Eficacia } \\
\text { Política } \\
\text { Externa }\end{array}$ & $\begin{array}{c}\text { Eficacia } \\
\text { Política } \\
\text { Interna }\end{array}$ & $\begin{array}{c}\text { Eficacia } \\
\text { Política } \\
\text { Externa }\end{array}$ \\
\hline Educación & $0.18^{* *}$ & $-0.09^{* *}$ & - & - \\
\hline Interés en la Política & $0.28^{* *}$ & -0.01 & $0.32^{* *}$ & -0.40 \\
\hline $\begin{array}{c}\text { Participación Política } \\
\text { Convencional }\end{array}$ & $0.34^{* *}$ & $-0.07^{*}$ & $0.41^{* *}$ & $-0.14^{* *}$ \\
\hline Confianza en el Gobierno & $0.08^{* *}$ & $0.42^{* *}$ & -0.82 & $0.15^{* *}$ \\
\hline $\begin{array}{c}\text { Satisfacción con la } \\
\text { Democracia }\end{array}$ & -0.03 & $0.46^{* *}$ & 0.01 & $0.16^{* *}$ \\
\hline
\end{tabular}

Nota: $* \mathrm{p}<0.05(5 \%) ; *$ p $<0.01(1 \%)$. Prueba de dos colas

Fuente: Datos recolectados por el GIPS-UPS, Quito - Ecuador (marzo 2017 y julio 2017). Elaboración propia.

La eficacia política interna se correlaciona de forma positiva y significativa con el nivel de educación, el interés en la política y la participación política convencional. Esto sugiere que las personas con mayor escolaridad e interés por las cuestiones políticas, tienden a su vez a sentirse más competentes política-mente. Del mismo modo, aquellos que se sienten internamente más eficaces para

${ }^{7}$ Dado que los índices de eficacia política interna y externa se calculan adicionando los puntajes-ítem de cada subescala, resulta procedente emplear el coeficiente $r$ de Pearson, puesto que los índices aditivos cuentan una métrica más continua (Ruiz et al., 2010). 
la política están más propensos a involucrarse en actividades políticas de tipo convencional. En contraste, la eficacia política externa presenta una asociación positiva y significativa con la confianza en el gobierno y la satisfacción con la democracia. Por lo tanto, aquellas personas que perciben una mayor receptividad del sistema político tienden a confiar más en el gobierno y estar más satisfechas con el funcionamiento de la democracia. Estos resultados se manifiestan tanto en $n_{1}$ como $n_{2}$, siendo compatibles con las expectativas teóricas planteadas inicialmente

Los datos también sugieren una conexión negativa entre eficacia política externa y variables como el grado de escolaridad o la participación política convencional. En el caso del grado de escolaridad, parecería que quienes tienen un mayor nivel educativo tienden a percibir una menor apertura de las autoridades públicas o los representantes políticos hacia las demandas ciudadanas. Desde una mirada más contextualizada, el estudio efectuado por Monsiváis-Carrillo (2017), a partir de una muestra de países latinoamericanos (incluyendo Ecuador), revela que son las personas con mayor educación formal quienes perciben de modo más crítico la calidad de la representación del gobierno y el sistema político. A su vez, esto explicaría el signo negativo de la relación observada entre eficacia interna y externa en el AFC. Por otra parte, aquellos que perciben una menor receptividad del sistema político tienden a participar más frecuentemente en actividades políticas convencionales, lo cual coincidiría con la tesis de que una mayor satisfacción con el gobierno, las instituciones políticas y la situación en general puede terminar inhibiendo la participación política de la ciudadanía (Amnå et al., 2004; Ribeiro et al., 2011). Considerando las particularidades del caso ecuatoriano, podría agregarse que el periodo correísta no ofreció condiciones favorables para la protesta social, criminalizándola y castigándola severamente (BasabeSerrano y Martínez, 2014). Por lo tanto, la insatisfacción con la responsividad de la clase política debió canalizarse mayoritariamente a través de los mecanismos participativos establecidos por el Estado, por vías legales y pacíficas.

Sin embargo, dado que estas correlaciones negativas son relativamente débiles, se requieren pruebas adicionales para constatar la existencia efectiva de dichas asociaciones. Situación similar ocurre con la posible conexión entre confianza en el gobierno y eficacia política interna, misma que se desvanece al limitar el análisis a estudiantes universitarios. En resumen, se verifica que cada tipo de eficacia política mantiene patrones diferenciados de asociación con el resto de variables de interés para la investigación sociopolítica. Esto constituye un indicador de la calidad del instrumento, pues los ítems propuestos son capaces de captar empíricamente las distinciones propuestas a nivel conceptual entre eficacia política interna y externa.

\section{d) Invarianza de Medida de la Escala de Eficacia Política}

La evaluación de invarianza de medida consiste en verificar si un instrumento puede conservar sus propiedades psicométricas entre diferentes segmentos sociales o entornos culturales, siendo capaz de medir el mismo constructo a partir de una estructura factorial idéntica independientemente del grupo de personas 
que se analice (Milfont y Fischer, 2010; Van de Schoot, Lugtig y Hox, 2012). El análisis de equivalencia de medida de la escala de eficacia política se concentró en la fase inicial de invarianza factorial ${ }^{8}$, misma que incluye pruebas de invarianza configural e invarianza métrica (Cheung y Rensvold, 1999).

La invarianza configural se obtiene cuando la estructura factorial del instrumento se conserva entre los grupos analizados, esto significa que en cada grupo se conceptualiza el constructo de interés de la misma forma, haciendo distinciones idénticas entre las dimensiones que lo componen. Por lo tanto, se espera que los ítems reflejen los mismos factores latentes en cada grupo (Barrera-Barrera, Navarro-García y Peris-Ortiz, 2015; Cheung y Rensvold, 2002; Gregorich, 2006). La invarianza métrica hace referencia a la equivalencia de las cargas factoriales entre grupos, es decir, los ítems se conectan con la misma intensidad a sus respectivos factores latentes. Esto indica que los grupos atribuyen el mismo significado a cada dimensión del constructo, lo cual permite que se manifiesten con la misma intensidad a través de sus respectivos indicadores observables (Gregorich, 2006; Milfont y Fischer, 2010; Van de Schoot et al., 2012).

Para probar los tipos de invarianza expuestos se procedió a efectuar un Análisis Factorial Confirmatorio Multigrupo (AFCMG) sobre las dos muestras independientes analizadas en este estudio. La estrategia de análisis para la invarianza de medida se basa en la construcción de una secuencia de modelos cada vez más restrictivos en relación con la estimación de parámetros. Cada modelo se construye a partir del anterior, añadiendo un número adicional de constricciones que reducen la cantidad de parámetros a estimar y aumentan los grados de libertad. Este tipo de sucesión entre modelos se conoce como modelos anidados (Milfont y Fischer, 2010). En este sentido, se elaboraron dos modelos anidados, el modelo 1 adopta invarianza configural, mientras que el modelo 2 agrega invarianza métrica. Estos modelos fueron puestos a prueba en términos de su bondad de ajuste a los datos. En el modelo 1 (invarianza configural) se mantuvo la misma estructura factorial (basada en los dos tipos de eficacia política) en cada muestra de estudio, con una estimación simultánea de parámetros para los dos grupos. El modelo 2 (invarianza métrica) tomó como punto de partida al modelo 1 y agregó la siguiente restricción: se asumió que todas las cargas factoriales son iguales en cada muestra analizada. Dada esta constricción, se estiman nuevamente los parámetros de modo simultáneo. Se espera que el ajuste de datos del modelo 1 sea satisfactorio, y que este ajuste no experimente un deterioro significativo al pasar al modelo 2 (Barrera-Barrera et al., 2015; Byrne, 2010; Cheung y Rensvold, 2002; Elosua, 2005; Vecchione et al, 2014).

Dado que las muestras de estudio cuentan con un desbalance importante ( $n_{1}=1136$ y $n_{2}=540$ ) se procedió a equipararlas para evitar distorsiones en los resultados del AFCMG, siguiendo las recomendaciones de Yoon y Lai (2018). Para ello se obtuvo un submuestra aleatoria de $n_{1}$ mediante el software SPSS 23, ajustando su tamaño en 540 casos al igual que $n_{2}$. Para evaluar las diferencias

${ }^{8}$ Etapas más avanzadas del análisis de invarianza de medida se dificultan debido al uso del método ADF para la estimación de parámetros en el AFC. 
entre los modelos anidados se empleó la prueba de diferencias de chi-cuadrado $(\Delta \times 2)$ con un nivel de significación de 0.05 , así como las diferencias en el índice de bondad de ajuste comparativo $(\Delta \mathrm{CFI})$ y en la raíz del residuo cuadrático promedio de aproximación ( $\triangle$ RMSEA). Los criterios de referencia para concluir la existencia de invarianza en la bondad de ajuste de los datos son los siguientes: $\Delta$ CFI $<-0.01, \Delta$ RMSEA $<0.015$ (Cheung y Rensvold, 2002; Elosua, 2005; Vecchione et al, 2014). Los resultados del AFCMG se presentan en la tabla 5.

Tabla 5. Prueba de invarianza configural y métrica de la escala de eficacia política

\begin{tabular}{|l|c|c|c|c|c|c|}
\hline Modelos de Prueba & $\mathrm{X} 2$ & $\mathrm{gl}$ & $\mathrm{p}$-valor & CFI & RMSEA & $\begin{array}{c}\text { Comparación } \\
\text { de modelos }\end{array}$ \\
\hline $\begin{array}{l}\text { Modelo 1: } \\
\text { Invarianza } \\
\text { Configural }\end{array}$ & 54.03 & 34 & 0.016 & 0.976 & 0.023 & \\
\hline $\begin{array}{l}\text { Modelo 2: } \\
\text { Invarianza Métrica }\end{array}$ & 63.03 & 40 & 0.012 & 0.972 & 0.023 & 2 vs 1 \\
\hline
\end{tabular}

\begin{tabular}{|l|c|c|c|c|c|}
\hline Modelos de Prueba & $\Delta \mathrm{X} 2$ & $\Delta \mathrm{gl}$ & $\mathrm{p}$-valor & $\Delta \mathrm{CFI}$ & $\Delta \mathrm{RMSEA}$ \\
\hline $\begin{array}{l}\text { Modelo 1: } \\
\text { Invarianza } \\
\text { Configural }\end{array}$ & & & & & \\
\hline $\begin{array}{l}\text { Modelo 2: } \\
\text { Invarianza Métrica }\end{array}$ & 9.00 & 6 & 0.173 & -0.004 & 0.000 \\
\hline
\end{tabular}

Fuente: Datos recolectados por el GIPS-UPS, Quito - Ecuador (marzo 2017 y julio 2017). Elaboración propia.

En el caso del modelo 1, el valor adoptado por los indicadores CFI ( $>0.95)$ y RMSEA $(<0.08)$ permite constatar la existencia de invarianza configural, esto implica que la estructura factorial de la escala de eficacia política se conserva entre las muestras de estudio. En otras palabras, cada grupo analizado distingue de 
forma equivalente entre eficacia política interna y externa a través de los ítems del instrumento. La invarianza métrica también puede verificarse, puesto que de acuerdo con la prueba de diferencias de chi-cuadrado no existen diferencias estadísticamente significativas entre el modelo 1 y el modelo $2\left(\Delta X^{2}=9 ; p=\right.$ 0.17). Asimismo, las diferencias registradas por los indicadores CFI y RMSEA se encuentran dentro de los criterios de referencia $(\triangle C F I=-0.004 ; \triangle R M S E A=$ 0.000). Esto significa que cada grupo concibe a la dimensión interna y externa de la eficacia política de forma equivalente, haciendo posible que los ítems de cada subescala sean capaces de medir con la misma precisión el comportamiento de sus respectivos factores latentes. Es decir, cada ítem recibe una interpretación análoga por parte de los integrantes de ambos grupos. Los resultados presentados permiten concluir que el instrumento propuesto cuenta con propiedades de invarianza factorial, reforzando su validez y confiabilidad como modelo de medición del sentido de eficacia política.

\section{DISCUSIÓN}

El sentido de eficacia política es un concepto explicativo fundamental para la comprensión del comportamiento político individual y colectivo, siendo necesario asegurar procedimientos consistentes y comparables de medición que faciliten una lectura precisa de su funcionamiento, tomando en cuenta las particularidades de sus dimensiones constitutivas y su aplicabilidad a contextos hispanoparlantes. Las pruebas estadísticas efectuadas en el presente trabajo proporcionan evidencia empírica a favor de la escala propuesta para la medición del sentido de eficacia política individual. Las propiedades psicométricas de validez de constructo, consistencia interna, validez externa e invarianza factorial han podido verificarse satisfactoriamente a partir del uso de dos muestras independientes extraídas de la realidad ecuatoriana en momentos pre y post electorales respectivamente. En vista de que momentos como las elecciones presidenciales tienen capacidad para producir cambios en las actitudes políticas de la ciudadanía (Banducci y Karp, 2003), la estrategia de recolección de datos tuvo entre sus fines contemplar estas posibles variaciones a la hora de probar la validez y fiabilidad del instrumento.

Los resultados expuestos permiten establecer dos hallazgos fundamentales: primero, la escala formulada por Niemi et al. (1991) para la medición de la eficacia política interna sugiere un grado aceptable de replicabilidad en contextos hispanoparlantes, siempre que el proceso de adaptación y traducción conserve el contenido esencial de sus ítems. Esta conclusión se desprende del buen desempeño psicométrico constatado para un contexto como el ecuatoriano, que si bien se configura a partir de un momento histórico que conjuga marcadas especificidades de carácter político, económico y social (descritas inicialmente), no deja de compartir similitudes con el resto de países latinoamericanos. Dado que dicha escala se desarrolló originalmente para otro tipo de contextos, los resultados aportan evidencia de validez intercultural. Segundo, los ítems desarrollados para 
la medición de la eficacia política externa cuentan con cualidades psicométricas de partida que resultan prometedoras de cara a estudios comprobatorios de mayor escala que puedan efectuarse a futuro.

El instrumento propuesto, suma de la adaptación de la escala de eficacia política interna de Niemi et al. (1991) y la construcción de una escala de eficacia política externa, pretende ser una contribución metodológica hacia una medición más estandarizada del sentido de eficacia política en el marco de los crecientes esfuerzos de investigación centrados en entornos hispanoparlantes. Aspectos como una estructura factorial coherente, confiabilidad de los ítems que componen cada subescala y evidencia de validez nomológica acorde con la literatura internacional, sugieren una correcta adaptación cultural de la métrica, ofreciendo perspectivas alentadoras de su aplicabilidad en el resto de países latinoamericanos y potencialmente en España. En este sentido, aunque la invarianza de medida del instrumento solo pudo verificarse en términos de invarianza factorial, esto constituye un prerrequisito necesario para la posterior realización de comparaciones interculturales que posibiliten la depuración y el logro de una escala de eficacia política consistente entre países (Cheung y Rensvold, 1999).

\subsection{Limitaciones $y$ futuras investigaciones}

El presente trabajo cuenta con ciertas limitaciones relativas al diseño muestral y el método de estimación en AFC. Las muestras empleadas proporcionaron información circunscrita al DMQ, tanto en lo referente a población general como universitaria, razón por la cual se recomienda realizar estudios con muestras representativas a nivel nacional, con el objetivo de contrastar los resultados empíricos aquí presentados y avanzar hacia evaluaciones de la aplicabilidad del instrumento entre diferentes países hispanoparlantes, tomando en consideración el ajuste cultural y las diferencias de contexto político. Por otra parte, la escala de valoración de los ítems tipo Likert empleados para medir la eficacia política interna y externa se limitó al uso de 4 opciones de respuesta, aspecto que restringió el uso de métodos de estimación más potentes en el AFC (por ejemplo, Máxima Verosimilitud), puesto que los ítems se trataron como politómicos. Ante esto, se sugiere que en posteriores aplicaciones se utilicen escalas tipo Likert con al menos 5 categorías de respuesta, lo cual además de facilitar la estimación de parámetros por medio de técnicas más potentes, posibilita un análisis más completo de la invarianza de medida del instrumento, pudiendo incluirse las siguientes pruebas de invarianza: escalar, varianza de error, varianza factorial, covarianza factorial y medias factoriales (Milfont y Fischer, 2010). Estas pruebas estadísticas adicionales resultan necesarias para el análisis de invarianza de medida entre diferentes entornos culturales y políticos. De este modo, la evaluación de invarianza cultural permitiría, de ser necesario, introducir ajustes en los ítems de la escala de eficacia política con el propósito de disponer de un instrumento lo suficientemente consistente y estandarizado entre países, mejorando la comparabilidad de la información y los resultados de investigación, fortaleciendo la 
comprensión de los fenómenos políticos e incluso abriendo la posibilidad del uso futuro de dicha escala en encuestas de opinión pública de aplicación regular a nivel de América Latina y España.

\section{BIBLIOGRAFÍA}

ACOCK, A. C. y CLARKE, H. D. (1990): “Alternative measures of political efficacy: Models and means”, Quality and Quantity, 24(1), pp. 87-105. Disponible en: https:// doi.org/10.1007/BF00221386

ACOCK, A., CLARKE, H. D. y STEWART, M. C. (1985): “A new model for old measures: A covariance structure analysis of political efficacy", The Journal of Politics, 47(4), pp. 1062-1084. Disponible en: https://doi.org/10.2307/2130807

AISH, A. M. y JÖRESKOG, K. G. (1990): “A panel model for political efficacy and responsiveness: An application of LISREL 7 with weighted least squares", Quality and Quantity, 24(4), pp. 405-426. Disponible en: https://doi.org/10.1007/ BF00152013

ÁLVAREZ, V. P. (2016): “De Correa al 2017: claves para entender el preludio electoral en Ecuador", Revista Política Latinoamericana, 2, pp. 1-11.

AMNÅ, E., MUNCK, I. y ZETTERBERG, P. (2004): "Meaningful participation? Political efficacy of adolescents in 24 countries", European Consortium of Political Research Joint Sessions, Uppsala, April 2004.

ANDUIZA, E., CANTIJOCH, M., GALLEGO, A. y SALCEDO, J. (2010): "Internet y participación política en España”, Opiniones y Actitudes, 63, pp. 1-67. Disponible en: http://www.cis.es/cis/export/sites/default/-Archivos/Publicaciones/OyA/ OyA63a.pdf

BALCH, G. I. (1974): "Multiple Indicators in Survey Research: The Concept "Sense of Political Efficacy"', Political Methodology, 1 (2), pp. 1-43.

BANDUCCI, S. A. y KARP, J. A. (2003): "How elections change the way citizens view the political system: campaigns, media effects and electoral outcomes in comparative perspective", British Journal of Political Science, 33(3), pp. 443-467. Disponible en: https://doi.org/10.1017/S000712340300019X

BANDURA, A. (1997): Self-efficacy: The exercise of control, New York, Freeman.

BARRERA-BARRERA, R., NAVARRO-GARCÍA, A. \& PERIS-ORTIZ, M. (2015): "El papel de la invarianza factorial en la validación del constructo calidad de servicio electrónico", Revista Europea de Dirección y Economía de la Empresa, 24(1), pp. 13-24. Disponible en: https://doi.org/10.1016/j.redee.2014.07.001

BASABE-SERRANO, S. y MARTÍNEZ, J. (2014): "Ecuador: Cada vez menos democracia, cada vez más autoritarismo... con elecciones", Revista de ciencia política, 34(1), pp. 145-170. Disponible en: http://dx.doi.org/10.4067/S0718090X2014000100007

BATISTA-FOGUET, J. M., COENDERS, G. y ALONSO, J. (2004): “Análisis factorial confirmatorio. Su utilidad en la validación de cuestionarios relacionados con la salud", Medicina clínica, 122(1), pp. 21-27.

BORJA, S. (2017): Los tentáculos de Odebrecht tocan la campaña electoral, disponible en http://elecciones2017.gkillcity.com/2017/02/06/odebrecht-ecuador-tocan-lacampana-electoral-ecuador/ [consulta: 29-11-2019] 
BRUSSINO, S., MEDRANO, L., SORRIBAS, P. y RABBIA, H. H. (2011): "Young adults' knowledge of politics: Evaluating the role of socio-cognitive variables using structural equations", The Spanish journal of psychology, 14(1), pp. 183-194. Disponible en: https://doi.org/10.5209/rev_SJOP.2011.v14.n1.16

BRUSSINO, S., RABBIA, H. H. y SORRIBAS, P. (2009): "Perfiles sociocognitivos de la participación política de los jóvenes”, Interamerican Journal of Psychology, 43(2), pp. 279-287.

BYRNE, B. M. (2010): Structural equation modeling with AMOS: Basic concepts, applications, and programming, New York, Routledge.

CAMPBELL, A., GURIN, G. y MILLER, W. (1954): The voter decides, Row Peterson, Evanstone.

CAPRARA, G. V., VECCHIONE, M., CAPANNA, C. Y MEBANE, M. (2009): "Perceived political self-efficacy: Theory, assessment, and applications", European Journal of Social Psychology, 39(6), pp. 1002-1020. Disponible en: http://dx.doi. org/10.1002/ejsp.604

CHALMERS, R. P. (2017): "On misconceptions and the limited usefulness of ordinal alpha", Educational and Psychological Measurement, pp. 1-16. Disponible en: https://doi.org/10.1177/0013164417727036

CHEUNG, G. W. y RENSVOLD, R. B. (1999): “Testing factorial invariance across groups: A reconceptualization and proposed new method", Journal of management, 25(1), pp. 1-27. Disponible en: https://doi.org/10.1177/014920639902500101

CHEUNG, G. W. y RENSVOLD, R. B. (2002): "Evaluating goodness-of-fit indexes for testing measurement invariance", Structural equation modeling, 9(2), pp. 233-255. Disponible en: https://doi.org/10.1207/S15328007SEM0902_5

CLENCH-AAS, J., NES, R. B., DALGARD, O. S. y AARØ, L. E. (2011): "Dimensionality and measurement invariance in the Satisfaction with Life Scale in Norway", Quality of Life Research, 20(8), pp. 1307-1317. Disponible en: https://doi. org/10.1007/s11136-011-9859-X

CRAIG, S. C. y MAGGIOTTO, M. A. (1982): "Measuring political efficacy". Political Methodology, 8 (3), pp. 85-109.

CRAIG, S. C., NIEMI, R. G. y SILVER, G. E. (1990): "Political efficacy and trust: A report on the NES pilot study ítems", Political behavior, 12(3), pp. 289-314. Disponible en: https://doi.org/10.1007/BF00992337

CUPANI, M. (2012): “Análisis de Ecuaciones Estructurales: conceptos, etapas de desarrollo y un ejemplo de aplicación", Revista Tesis, 2 (1), pp. 186-199.

DE LA CRUZ, G. y VALENZUELA. R. (2019): "Estrategia discursiva anti statu quo en la transformación del sistema de partidos en tres países de América Latina", Studia Politicæ, 46, pp. 137-168.

DE MARCO, S. Y ROBLES MORALES, J. M. (2012): “Uso de los blogs políticos: análisis de algunos factores determinantes", Arbor, 188(756), pp. 689-705. Disponible en: https://doi.org/10.3989/arbor.2012.756n4005

ELOSUA OLIDEN, P. \& ZUMBO, B. D. (2008): “Coeficientes de fiabilidad para escalas de respuesta categórica ordenada", Psicothema, 20(4), pp. 896-901.

ELOSUA, P. (2005): "Evaluación progresiva de la invarianza factorial entre las versiones original y adaptada de una escala de autoconcepto", Psicothema, 17(2), pp. 356362.

FINKEL, S. E. (1985): "Reciprocal effects of participation and political efficacy: A panel analysis", American Journal of political science, 29 (4), pp. 891-913. 
GIL DE ZÚÑIGA, H., MOLYNEUX, L. y ZHENG, P. (2014): "Social media, political expression, and political participation: Panel analysis of lagged and concurrent relationships", Journal of Communication, 64(4), pp. 612-634. Disponible en: https:// doi.org/10.1111/jcom.12103

GONZÁLEZ ALONSO, J. \& PAZMIÑO SANTACRUZ, M. (2015): “Cálculo e interpretación del Alfa de Cronbach para el caso de validación de la consistencia interna de un cuestionario, con dos posibles escalas tipo Likert”, Revista Publicando, 2(1), pp. 62-67.

GREGORICH, S. E. (2006): "Do self-report instruments allow meaningful comparisons across diverse population groups? Testing measurement invariance using the confirmatory factor analysis framework", Medical care, 44(11), pp. S78-S94. Disponible en: http://dx.doi.org/10.1097/01.mlr.0000245454.12228.8f

GROSSI, J. y OVEJERO, A. (1994): “Alienación y participación política en la Universidad de Oviedo”, Psicología Política, (8), pp. 45-62.

HAYES, B. C. y BEAN, C. S. (1993): "Political efficacy: a comparative study of the United States, West Germany, Great Britain and Australia", European Journal of Political Research, 23(3), pp. 261-280. Disponible en: http://dx.doi. org/10.1111/j.1475-6765.1993.tb00359.x

HEISS, R. y MATTHES, J. (2016): "Mobilizing for some: The effects of politicians' participatory Facebook posts on young people's political efficacy", Journal of Media Psychology: Theories, Methods, and Applications, 28(3), pp. 123-135. Disponible en: https://doi.org/10.1027/1864-1105/a000199

HELAL, A. M. y HAMZA, E. G. A. (2015): "Political Efficacy, Voting Behavior and Partisanship among University Students", International Journal of Humanities and Social Science, 5 (9), pp. 300-315.

IMHOFF, D., GUTIERREZ, Y. y BRUSSINO, S. (2009): “Jóvenes y ciudadanía: indagación acerca del modo de ejercicio de la ciudadanía de los los/as jóvenes a partir de la relación de las orientaciones psicológicas de la politización juvenil con la participación política", Revista Tesis, 1(1), pp. 21-38.

JENNINGS, M. y ZEITNER, V. (2003): "Internet use and civic engagement: A longitudinal analysis", Public Opinion Quarterly, 67(3), pp. 311-334. Disponible en: https:// doi.org/10.1086/376947

JIANG, L. (2016): "The effects of the Internet on online and offline political participation among citizens in Australia", 66 th Annual International Conference of British Political Science Association, Brighton, United Kingdom, 21 - 23 March 2016.

KÖLLN, A. K., ESAIASSON, P. y TURPER, S. (2013): "External efficacy and perceived responsiveness-same, same or different", Annual conference of the Elections, Public Opinion and Parties (EPOP) specialist group, pp. 13-15.

LABARTHE, S. y SAINT-UPÉRY, M. (2017): "Leninismo versus correísmo: la «tercera vuelta» en Ecuador", Nueva Sociedad, 272, pp. 29-42.

LATINOBARÓMETRO (2016): Latinobarómetro 2016: banco de datos, disponible en: http://www.latinobarometro.org/latOnline.jsp [consulta: 20-11-2019]

LIANG, J., ASANO, H., BOLLEN, K. A., KAHANA, E. F. y MAEDA, D. (1987): "Cross-cultural comparability of the Philadelphia geriatric center morale scale: An American-Japanese comparison”, Journal of Gerontology, 42(1), pp. 37-43. Disponible en: https://doi.org/10.1093/geronj/42.1.37 
LLORET-SEGURA, S., FERRERES-TRAVER, A., HERNÁNDEZ-BAEZA, A. y TOMÁS-MARCO, I. (2014): "El análisis factorial exploratorio de los ítems: una guía práctica, revisada y actualizada”, Anales de psicología, 30(3), pp. 1151-1169. Disponible en: http://dx.doi.org/10.6018/analesps.30.3.199361

MCPHERSON, J. M., WELCH, S. y CLARK, C. (1977): "The stability and reliability of political efficacy: Using path analysis to test alternative models", American Political Science Review, 71(2), pp. 509-521. Disponible en: https://doi.org/10.1017/ S0003055400267427

MELÉNDEZ, C. y MONCAGATTA, P. (2017): "Ecuador: Una década de correísmo", Revista de Ciencia Política, 37(2), pp. 413-448. Disponible en: http://dx.doi. org/10.4067/s0718-090x2017000200413

MILFONT, T. L. y FISCHER, R. (2010): “Testing measurement invariance across groups: Applications in crosscultural research", International Journal of psychological research, 3(1), pp. 111-130. Disponible en: http://dx.doi. org/10.21500/20112084.857

MILOSEVI区-ĐORĐEVI区, J. S. y ŽEŽELJ, I. L. (2017): “Civic activism online: Making young people dormant or more active in real life?", Computers in Human Behavior, 70, pp. 113-118. Disponible en: https://doi.org/10.1016/j.chb.2016.12.070

MIRANDA-ZAPATA, E., RIQUELME-MELLA, E., CIFUENTES-CID, H. y RIQUELME-BRAVO, P. (2014): “Análisis factorial confirmatorio de la Escala de habilidades sociales en universitarios chilenos", Revista Latinoamericana de Psicología, 46(2), pp. 73-82. Disponible en: https://doi.org/10.1016/S0120-0534(14)70010-X

MONSIVAIS-CARRILLO, A. (2017): "La desafección representativa en América Latina", Andamios, 14(35), pp. 17-41.

MOREIRA, C. (2017): "El largo ciclo del progresismo latinoamericano y su freno: los cambios políticos en América Latina de la última década (2003-2015)", Revista Brasileira de Ciencias Sociais, 32(93), pp. 1-28. Disponible en: http://dx.doi. org/10.17666/329311/2017

MORRELL, M. E. (2003): "Survey and experimental evidence for a reliable and valid measure of internal political efficacy", The Public Opinion Quarterly, 67(4), pp. 589-602.

MUÑOZ-JARAMILLO, F. (2014): Balance crítico del gobierno de Rafael Correa, Quito, Universidad Central del Ecuador.

NIEMI, R. G., CRAIG, S. C. y MATTEI, F. (1991): "Measuring internal political efficacy in the 1988 National Election Study", American Political Science Review, 85(4), pp. 1407-1413. Disponible en: https://doi.org/10.2307/1963953

NUVIALA NUVIALA, A., GRAO-CRUCES, A., TEVA-VILLÉN, M. R., PÉREZORDÁS, R., GARCÍA-FERNÁNDEZ, J. y BERNAL-GARCÍA, A. (2014): “Adaptación y validación de la Escala de Intenciones Futuras de Comportamiento en usuarios de servicios deportivos", Universitas Psychologica, 13(3), pp. 1071-1082. Disponible en: http://dx.doi.org/10.11144/Javeriana.UPSY13-3.avei

OLSSON, U. H., FOSS, T. y TROYE, S. V. (2003): "Does the ADF fit function decrease when the kurtosis increases?", British Journal of Mathematical and Statistical Psychology, 56(2), pp. 289-303. Disponible en: http://dx.doi. org/10.1348/000711003770480057

OVIEDO, H. C. y ARIAS, A. C. (2005): “Aproximación al uso del coeficiente alfa de Cronbach", Revista colombiana de psiquiatría, 34(4), pp. 572-580. 
PACHANO, S. (2008): “Calidad de la democracia y colapso del sistema de partidos en Ecuador", Seminario Partidos políticos y calidad de la democracia, México, Noviembre 2008.

PACHANO, S. (2017): "Elecciones y fin de ciclo en Ecuador", $9^{\circ}$ Congreso Latinoamericano de Ciencia Política organizado por la Asociación Latinoamericana de Ciencia Política (ALACIP), Montevideo, Julio 2017.

RIBEIRO, E. A., BORBA, J. y SALINAS, A. (2011): "Participación y democracia en América Latina: los determinantes individuales de la participación política", Foro Internacional, 51 (2), pp. 242-270.

RUIZ, M. A., PARDO, A. y SAN MARTÍN, R. (2010): "Modelos de ecuaciones estructurales", Papeles del psicólogo, 31(1), pp. 34-45.

SCHERMELLEH-ENGEL, K., MOOSBRUGGER, H. y MÜLLER, H. (2003): "Evaluating the fit of structural equation models: Tests of significance and descriptive goodness-of-fit measures", Methods of psychological research online, 8(2), pp. 23 74.

SCHEUFELE, D. A. y NISBET, M. C. (2002): "Being a citizen online: New opportunities and dead ends", Harvard International Journal of Press/Politics, 7(3), pp. 55-75. Disponible en: http://journals.sagepub.com/doi/pdf/10.1177/1081180X0200700304

SOMUANO VENTURA, M. F. (2005): "Más allá del voto: modos de participación política no electoral en México", Foro Internacional, 45 (1), pp. 65-88.

SORRIBAS, P. M. y BRUSSINO, S. A. (2013): "La participación política orientada al sistema representativo: dimensiones y factores explicativos", Psicología Política, (47), pp. 91-112.

VALENTINO, N. A., BRADER, T., GROENENDYK, E. W., GREGOROWICZ, K. y HUTCHINGS, V. L. (2011): "Election night's alright for fighting: The role of emotions in political participation", The Journal of Politics, 73(1), pp. 156-170. Disponible en: https://doi.org/10.1017/S0022381610000939

VAN DE SCHOOT, R., LUGTIG, P. y HOX, J. (2012): "A checklist for testing measurement invariance", European Journal of Developmental Psychology, 9(4), pp. 486492. Disponible en: https://doi.org/10.1080/17405629.2012.686740

VECCHIONE, M., CAPRARA, G. V., CAPRARA, M. G., ALESSANDRI, G., TABERNERO, C. y GONZÁLEZ-CASTRO, J. L. (2014): “The Perceived Political SelfEfficacy Scale-Short Form (PPSE-S): A Validation Study in Three Mediterranean Countries", Cross-Cultural Research, 48(4), pp. 368-384. Disponible en: https://doi. org/10.1177/1069397114523924

WAGENMAKERS, E. J. y FARRELL, S. (2004): “AIC model selection using Akaike weights", Psychonomic bulletin \& review, 11(1), pp. 192-196. Disponible en: https://doi.org/10.3758/BF03206482

YANG, H. C. y DEHART, J. L. (2016): "Social media use and online political participation among college students during the US election 2012", Social Media+ Society, 2(1), pp. 1-18. Disponible en: http://doi.org/10.1177/2056305115623802

YOON, M. \& LAI, M. H. (2018): "Testing Factorial Invariance With Unbalanced Samples", Structural Equation Modeling: A Multidisciplinary Journal, 25(2), pp. 201213. Disponible en: https://doi.org/10.1080/10705511.2017.1387859

ZAPATA-MAFLA, S. y CUESTA, E. M. (2018): “Crisis, representación y populismo: Una mirada al caso de Ecuador”, Debates Latinoamericanos, 33, pp. 104-115. 
ZHANG, W., JOHNSON, T. J., SELTZER, T. y BICHARD, S. L. (2010): “The revolution will be networked: The influence of social networking sites on political attitudes and behavior", Social Science Computer Review, 28(1), pp. 75-92. Disponible en: https://doi.org/10.1177/0894439309335162

ZUMÁRRAGA ESPINOSA, M., CAROFILIS CEDEÑO, C. y REYES VALENZUELA, C. (2018): "El efecto movilizador de las emociones sobre la participación política online en la segunda vuelta de las elecciones presidenciales en Ecuador 2017”, Revista Democracias, 5, pp. 25-62.

ZUMÁRRAGA ESPINOSA, M., REYES VALENZUELA, C. y CAROFILIS CEDEÑO, C. (2017): “¿Verdad o ficción? El uso político de las redes sociales en la participación política offline en las elecciones presidenciales en Ecuador", Análisis Político, 30(91), pp. 130-145. Disponible en: https://doi.org/10.15446/anpol. v30n91.70268 